\title{
Utility of preoperative three-dimensional CT bronchography and angiography in uniportal video-assisted thoracoscopic anatomical lobectomy: a retrospective propensity score-matched analysis
}

\author{
Xin-Yu Zhu ${ }^{1,2 \#}$, Fei-Rong Yao ${ }^{3 \#}$, Chun $\mathrm{Xu}^{1,2}$, Cheng Ding ${ }^{1,2}$, Jun Chen $^{1,2}$, Wen-Yi Wang ${ }^{1,2}$, Liu-Ying Pan ${ }^{1,2}$, \\ Jun Zhao ${ }^{1,2}$, Chang $\mathrm{Li}^{1,2}$ \\ ${ }^{1}$ Department of Thoracic Surgery, The First Affiliated Hospital of Soochow University, Medical College of Soochow University, Suzhou, China; \\ ${ }^{2}$ Institute of Thoracic Surgery, The First Affiliated Hospital of Soochow University, Suzhou, China; ${ }^{3}$ Department of Radiology, The First Affiliated \\ Hospital of Soochow University, Medical College of Soochow University, Suzhou, China \\ Contributions: (I) Conception and design: XY Zhu, FR Yao, J Zhao, C Li; (II) Administrative support: J Zhao, C Li; (III) Provision of study \\ materials or patients: J Zhao, C Xu, C Ding, C Li; (IV) Collection and assembly of data: XY Zhu, FR Yao, LY Pan, J Chen; (V) Data analysis and \\ interpretation: XY Zhu, FR Yao, C Li; (VI) Manuscript writing: All authors; (VII) Final approval of manuscript: All authors. \\ \#These authors contributed equally to this work. \\ Correspondence to: Jun Zhao; Chang Li. Department of Thoracic Surgery, The First Affiliated Hospital of Soochow University, Medical College of \\ Soochow University, Suzhou 215000, China. Email: zhaojia0327@126.com; cli@suda.edu.cn.
}

Background: Personalized three-dimensional (3D) reconstruction can help surgeons to overcome technical challenges and variations of pulmonary anatomic structures in the performance of uniportal video-assisted thoracoscopic surgery (UVATS), thus improving the safety and efficacy of the procedure. This study aims to evaluate the utility of preoperative 3D-CT bronchography and angiography (3D-CTBA) with Exoview software in the assessment of anatomical variations of pulmonary vessels, and to analyze short-term surgical outcomes in patients undergoing UVATS lobectomy.

Methods: We retrospectively analyzed the data of 198 consecutive patients who underwent curative UVATS lobectomy between November 2019 and September 2020. The patients were divided into an "Exoview" group (n=53) and a "non-Exoview" group ( $\mathrm{n}=145)$. We performed 1:1 propensity score matching and compared intraoperative and postoperative outcomes between the two groups. A subgroup analysis of 74 patients who underwent single-direction uniportal lobectomy was also conducted. Aberrant pulmonary vessel patterns related to the surgery were also examined.

Results: The operative time in the Exoview group was significantly shorter than that in the non-Exoview group, both before $(145.7 \pm 33.9$ vs. $159.5 \pm 41.6$ minutes, $\mathrm{P}=0.032)$ and after $(145.7 \pm 33.9$ vs. $164.2 \pm 41.8$ minutes, $\mathrm{P}=0.014)$ propensity score matching. The number of mediastinal lymph nodes dissected was higher in the Exoview group than in the non-Exoview group $(8.19 \pm 6.89$ vs. $5.78 \pm 3.3, \mathrm{P}=0.024)$ after propensity score matching. Intraoperative blood loss showed a statistical difference between the Exoview and non-Exoview groups $(60.4 \pm 45.4$ vs. $100.8 \pm 83.9, \mathrm{P}=0.009)$. Four types of arterial variations and 2 types of venous variations related to the surgery were observed among 8 patients (15\%), which have rarely been reported before.

Conclusions: Personalized preoperative 3D-CT bronchography and angiography helped to clearly visualize the pulmonary anatomical structures and could contribute to the safe and efficient performance of UVATS anatomical lobectomy.

Keywords: Uniportal video-assisted thoracoscopic surgery (UVATS); three-dimensional computed tomography bronchography and angiography (3D-CTBA); single-direction lobectomy

Submitted Jan 11, 2021. Accepted for publication Mar 03, 2021.

doi: $10.21037 /$ atm-21-474

View this article at: http://dx.doi.org/10.21037/atm-21-474 


\section{Introduction}

Since it was first described in 2011, uniportal video-assisted thoracoscopic lobectomy has been increasingly applied in the treatment of lung cancer patients around the world, especially in China $(1,2)$. Compared to conventional multiportal VATS, uniportal video-assisted thoracoscopic surgery (UVATS) is reported to entail less surgical trauma, reduced postoperative pain, and a shorter hospital stay, and has acceptable mid-term survival outcomes (3), without compromising oncologic principles (4-6). Moreover, a single incision tends to be more acceptable to patients. However, due to the reductions in the number of operating ports and space, UVATS demands higher surgical skill and proficiency from surgeons and their assistants, especially in cases with incomplete lung fissures, anatomic variations of pulmonary vessels, and a large number of calcified lymph nodes (7-11), and can lead to serious complications, such as unexpected bleeding (12). Therefore, individual preoperative anatomical simulations and detailed planning of surgical procedures are conducive to the safety and feasibility of UVATS.

With the development of thin-section multidetector CT (MDCT) and application software technology, threedimensional (3D) reconstruction imaging has been widely adopted by surgeons. Compared with traditional two-dimensional (2D) CT imaging, 3D reconstruction imaging can provide physicians with a more detailed view of the relative position of the pulmonary anatomical structure $(13,14)$. To date, various $3 \mathrm{D}$ reconstruction software applications have been successfully applied by researchers to improve the precision of segmentectomy, subsegmentectomy, and even sub-subsegmentectomy (15-18). As for lobectomy, Zhang's study once investigated the perioperative outcomes and learning curve of singledirection UVATS right upper lobectomy assisted with 3D-CTBA (19). Few researches focused on all kinds of lobectomy regardless of tumor location or operation pathway. Therefore, studies on the application of imaging software in UVATS lobectomy are limited.

Exoview is a free and open-source $3 \mathrm{D}$ reconstruction software independently developed by our medical team. The efficient application of Exoview in VATS segmentectomy was described in detail in our previous study (13). In this retrospective study, we aimed to evaluate the utility of $3 \mathrm{D}$ CT bronchography and angiography with Exoview software in the preoperative assessment of anatomical variations of pulmonary vessels, and to analyze the effectiveness of this approach in improving short-term surgical outcomes in patients undergoing UVATS. We present the following article in accordance with the STROBE reporting checklist (available at http://dx.doi.org/10.21037/atm-21-474).

\section{Methods}

\section{Patients}

From November 2019 to September 2020, a total of 236 consecutive patients underwent anatomical lobectomy in The First Affiliated Hospital of Soochow University (Suzhou, China). Among them, 198 patients who received curative UVATS lobectomy were included in this retrospective study. All surgeries were performed by 3 experienced experts (J.Z., C.L., and C.X.) with experience of more than 500 cases of pulmonary anatomical resection. This retrospective study was approved by The First Affiliated Hospital of Soochow University (ethical approval no. 2020232). All procedures performed in this study involving human participants were in accordance with the Declaration of Helsinki (as revised in 2013). Individual consent for this retrospective analysis was waived. All data were treated anonymously to protect personal privacy.

The exclusion criteria were as follows: (I) patients with serious pleural adhesions; (II) patients who underwent complex UVATS procedures such as bilobectomy, pneumonectomy, sleeve resection with wedge resection of another lobe, or double-sleeve bronchovascular resection, or who had other organs requiring treatment; (III) patients whose operation needed to be performed by another surgical department. The flow chart for patient selection is illustrated in Figure 1.

\section{Preoperative CT and $3 D$ reconstruction}

\section{Exoview group}

Patients underwent CT pulmonary arteriography/ venography $(\mathrm{CTPA} / \mathrm{V})$ using a 64-slice multi-detector CT (MDCT) unit (Aquilion-64, Toshiba Medical Systems, Tokyo, Japan) with injection of contrast medium (iodixanol, Visipaque 320, GE Healthcare, Cork, Ireland) after admission. Image data in DICOM format were imported into the Exoview system to complete the 3D reconstruction, with pulmonary bronchi, arteries, veins, and tumors marked out with different colors (Figure 2). Detailed parameters and procedures have been reported in our previous article (13). For each individual, careful preoperative planning was carried out by surgeons on the basis of the $3 \mathrm{D}$ 


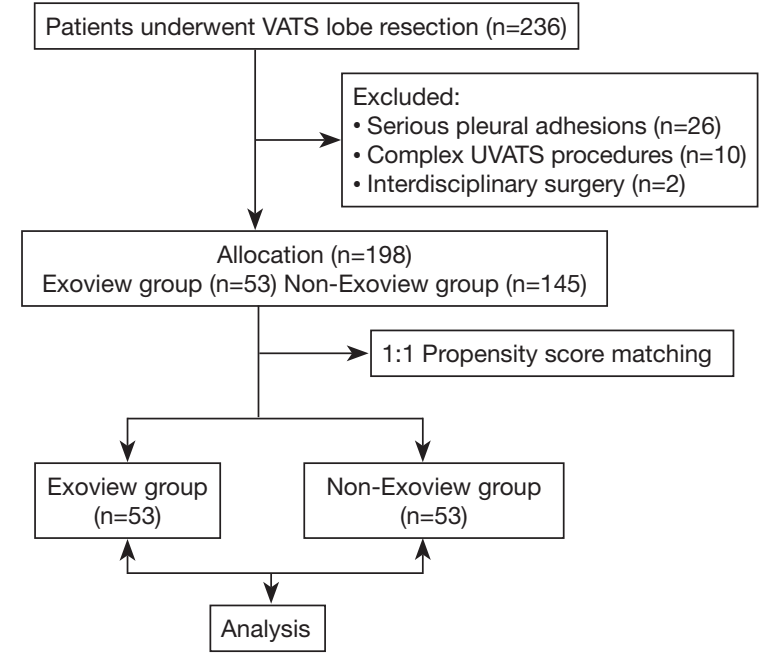

Figure 1 Flowchart of the study. UVATS, uniportal video-assisted thoracic surgery.

reconstruction images to reduce the risk of unplanned injury of aberrant anatomical structures. Additionally, we briefly defined several variations of pulmonary vessels.

\section{Non-Exoview group}

The reasons for which patients in the non-Exoview group did not undergo Exoview preoperative reconstruction were listed as follows: (I) the patient had undergone normal enhanced computed tomography shortly before admission; (II) the patient had a contraindication to contrast agent; (III) poor image quality; (IV) unplanned lobectomy. Target structures were identified by the same 3 experienced surgeons mentioned above on the basis of $2 \mathrm{D}$ thin-layer $\mathrm{CT}$ and their surgical experience. Other management methods and procedures were the same as those in the Exoview group.

\section{Surgical procedures}

All surgeries were performed under general anesthesia, with double-lumen endotracheal intubation and singlelung ventilation. Each patient was placed in the lateral decubitus position, with the healthy-side down. A single incision of approximately $3 \mathrm{~cm}$ in length was made along the anterior axillary line at the $4^{\text {th }}$ intercostal space for upper lobectomy and at the $5^{\text {th }}$ intercostal space for middle or lower lobectomy, without rib spreading. A plastic wound protector was used. A 10-mm, 30-degree thoracoscope (Karl Storz, Tuttlingen, Germany) was placed at the superior side of the incision, and several thoracoscopic instruments were concurrently attached to the single port beneath the thoracoscope. Single-direction thoracoscopic lobectomy was performed according to the completeness of lung fissures and the surgeon's habits. Single-direction lobectomy was defined as follows: first, the bronchi and vessels were managed at the root of the pulmonary structures in the hilum. The incomplete lung fissures were then manipulated with endoscopic staplers (Ethicon EndoSurgery Inc., Cincinnati, OH, USA) (20). All patients who were diagnosed as invasive carcinoma based on the results of intraoperative frozen section accepted systematic mediastinal lymph node dissection or sampling if conditions permitted.

\section{Data collection and statistical analysis}

Clinical data included patient age, sex, smoking and drinking history, body mass index (BMI), maximum lesion diameter, tumor location, pulmonary function, preoperative complications, postoperative pathology, operation characteristics (operative time, type of lymph node dissection, stations and numbers of dissected lymph nodes, incidence of conversion to multiport VATS or thoracotomy, and intraoperative blood loss), postoperative recovery (chest drainage, postoperative hospital stay, and postoperative complications), and anatomical variations of pulmonary vessels verified with the Exoview software. Postoperative complications were graded according to the Clavien-Dindo classification (21). Complications of grade I (representing minor complications requiring no treatment) were excluded. Patients' preoperative characteristics and surgical outcomes were collected from the HaiTai database (Nanjing, Jiangsu, China).

IBM SPSS Statistics (version 26.0, IBM Corp., Armonk, NY, USA) was used for all statistical analyses. Categorical data were presented as number (\%) and analyzed with the $\chi^{2}$ test or Fisher's exact test. Continuous variables were expressed as the mean \pm standard deviation (SD) and compared between groups using an independent 2 -samples $t$-test. Two-sided $\mathrm{P}<0.05$ was considered to be statistically significant.

To minimize the influence of selection bias and potential confounders, propensity score-matching (PSM) was performed to balance differences between groups. Propensity scores were calculated by a logistic regression model with variables including age, sex, BMI, maximal lesion size, type of surgical resection, and lymph node dissection. Patients in the Exoview and non-Exoview groups 

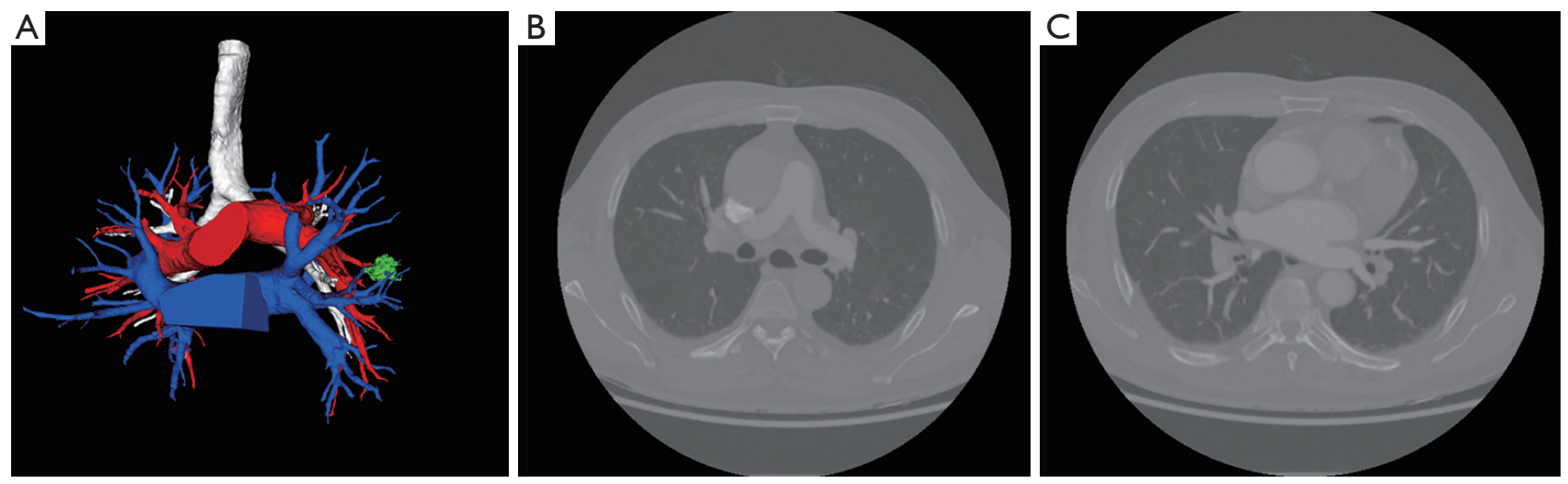

Figure 2 Preoperative lobectomy simulation according to the 3D-CTBA digital model and 2D-CTPA/V image. (A) In this patient, a 3D digital model was reconstructed by Exoview. The bronchi were marked in white. The pulmonary arteries were marked in red. The pulmonary veins were marked in blue. And the tumor was marked in green. (B) 2D-CTPA image of the same patient. Contrast medium was full of pulmonary arteries. (C) 2D-CTPV image of the same patient. Contrast medium was full of pulmonary veins. 3D-CTBA, threedimensional computed tomography bronchography and angiography; 2D-CTPA/V, two-dimensional computed tomography pulmonary arteriography/venography.

were matched at a ratio of 1:1 according to the propensity scores. A subgroup analysis of patients who underwent single-direction lobectomy was also performed.

\section{Results}

\section{General patient characteristics}

Between November 2019 and September 2020, a total of 236 patients accepted VATS anatomical lobectomy. After screening for eligibility, 198 patients (90 men and 108 women) who received curative UVATS lobectomy were enrolled in the analysis. Among them, 188 (95\%) had primary or metastatic lung cancer and $10(5 \%)$ had other benign lung diseases. Preoperative $3 \mathrm{D}$ reconstruction was performed for $53(27 \%)$ patients in the study cohort but reconstruction data was not available for 145 (73\%) patients for the reasons outlined above.

The general characteristics of the entire cohort $(\mathrm{N}=198)$ and those of patients after PSM $(\mathrm{N}=106)$ during the study period are summarized in Table 1 . The distribution of baseline characteristics was compared between the Exoview group and the non-Exoview group (Table 1). Before matching, patients who underwent $3 \mathrm{D}$ imaging preoperatively were shown to be more likely to receive a single-direction lobectomy than those who did not undergo $3 \mathrm{D}$ imaging (49.1\% vs. $33.1 \%, \mathrm{P}=0.04)$. There were no significant differences between the two groups with respect to sex, age, BMI, smoking history, other comorbidities (hypertension, diabetes mellitus, and coronary heart disease), pulmonary function [forced expiratory volume in $1 \mathrm{~s}$ (FEV1), percentile forced expiratory volume in $1 \mathrm{~s}$ (\%FEV1), and percentile maximal voluntary ventilation (\%MVV)], maximal tumor size on CT scan, or the location of pulmonary disease. After 1:1 PSM, 53 pairs of cases remained for analysis. All the demographic and clinical parameters were comparable between the Exoview group and non-Exoview group.

\section{Intraoperative and postoperative outcomes}

As illustrated in Table 2, the operative time in the Exoview group was significantly shorter than that in the nonExoview group both before $(145.7 \pm 33.9$ vs. $159.5 \pm 41.6 \mathrm{~min}$, $\mathrm{P}=0.032)$ and after $(145.7 \pm 33.9$ vs. $164.2 \pm 41.8 \mathrm{~min}, \mathrm{P}=0.014)$ PSM. Furthermore, significantly more mediastinal lymph nodes were resected in Exoview group than in the nonExoview group $(8.19 \pm 6.89$ vs. $5.78 \pm 3.3, \mathrm{P}=0.024)$ after PSM. However, there were no significant differences between the two groups in regard to other surgical characteristics. Shortterm postoperative recovery was similar in the two groups, and no 30-day postoperative mortality occurred in either group. 
Table 1 Clinical baseline characteristics of patients with and without preoperative 3D-CTBA before and after propensity score matching

\begin{tabular}{|c|c|c|c|c|c|c|}
\hline \multirow[b]{2}{*}{ Characteristics } & \multicolumn{3}{|c|}{ Entire cohort $(\mathrm{N}=198)$} & \multicolumn{3}{|c|}{ Propensity score matching ( $\mathrm{N}=106)$} \\
\hline & $\begin{array}{l}\text { Exoview group } \\
\qquad(\mathrm{N}=53)\end{array}$ & $\begin{array}{l}\text { Non-Exoview } \\
\text { group }(N=145)\end{array}$ & $P$ value & $\begin{array}{l}\text { Exoview group } \\
\qquad(\mathrm{N}=53)\end{array}$ & $\begin{array}{l}\text { Non-Exoview } \\
\text { group }(\mathrm{N}=53)\end{array}$ & $P$ value \\
\hline Male & $20(37.7)$ & $70(48.30)$ & & $20(37.7)$ & $22(41.5)$ & \\
\hline Female & $33(62.30)$ & $75(51.70)$ & & $33(62.30)$ & $31(58.5)$ & \\
\hline Body mass index, $\mathrm{kg} / \mathrm{m}^{2}$ & $23.9 \pm 2.8$ & $23.8 \pm 3.1$ & 0.684 & $23.9 \pm 2.8$ & $23.4 \pm 2.9$ & 0.301 \\
\hline Smoking history, n (\%) & & & 0.720 & & & 0.566 \\
\hline Never & $45(84.9)$ & $120(82.8)$ & & $45(84.9)$ & $47(88.7)$ & \\
\hline$\% \mathrm{MVV}, \%$ & $88.2 \pm 22.5$ & $89.1 \pm 20.7$ & 0.809 & $88.2 \pm 22.5$ & $88.8 \pm 18.4$ & 0.854 \\
\hline \multicolumn{7}{|l|}{ Comorbidity, n (\%) } \\
\hline Hypertension & $20(37.7)$ & $43(29.7)$ & 0.280 & $20(37.7)$ & $19(35.8)$ & 0.840 \\
\hline Diabetes mellitus & $3(5.7)$ & $10(6.9)$ & 1.000 & $3(5.7)$ & $4(7.5)$ & 1.000 \\
\hline Coronary heart disease & $8(15.1)$ & $20(13.8)$ & 0.816 & $8(15.1)$ & $12(22.6)$ & 0.321 \\
\hline Maximal lesion size, $\mathrm{mm}$ & $20.0 \pm 8.3$ & $22.1 \pm 14.1$ & 0.209 & $20.0 \pm 8.3$ & $17.5 \pm 7.8$ & 0.107 \\
\hline Single-direction approach, n (\%) & $26(49.1)$ & $48(33.1)$ & $0.040^{\star}$ & $26(49.1)$ & $21(39.6)$ & 0.328 \\
\hline Lower lobe & $20(37.7)$ & $55(37.9)$ & & $20(37.7)$ & $13(24.5)$ & \\
\hline $\begin{array}{l}\text { Mediastinal lymph node dissection or } \\
\text { sampling, } n(\%)\end{array}$ & $51(96.2)$ & $128(88.3)$ & 0.093 & $51(96.2)$ & $53(100)$ & 0.475 \\
\hline
\end{tabular}

*, $\mathrm{P}<0.05$. FEV1, forced expiratory volume in $1 \mathrm{~s}$; \%FEV1, percentile forced expiratory volume in $1 \mathrm{~s}$; \%MVV, percentile maximal voluntary ventilation.

Besides, a subgroup analysis of 74 patients who received a single-direction lobectomy was performed. The clinical baseline parameters of these patients are shown in Table 3 . Intraoperative blood loss showed a statistical difference between the Exoview group and non-Exoview group (60.4 \pm 45.4 vs. $100.8 \pm 83.9, \mathrm{P}=0.009$ ) (Table 4). However, there were no significant differences in other perioperative characteristics between the two groups.

\section{Anatomic variations verified with Exoview software}

In the evaluation of $3 \mathrm{D}$ reconstruction images of 53 patients, 4 types of arterial variations and 2 types of venous variations related to the surgery were observed among 8 patients (15\%), and these variations have rarely been reported before (Figures 3-8). All aberrant pulmonary artery $(\mathrm{PA})$ and pulmonary vein $(\mathrm{PV})$ branches were precisely confirmed by $3 \mathrm{D}$ reconstruction. 
Table 2 Postoperative parameters of patients with and without preoperative 3D-CTBA before and after propensity score matching

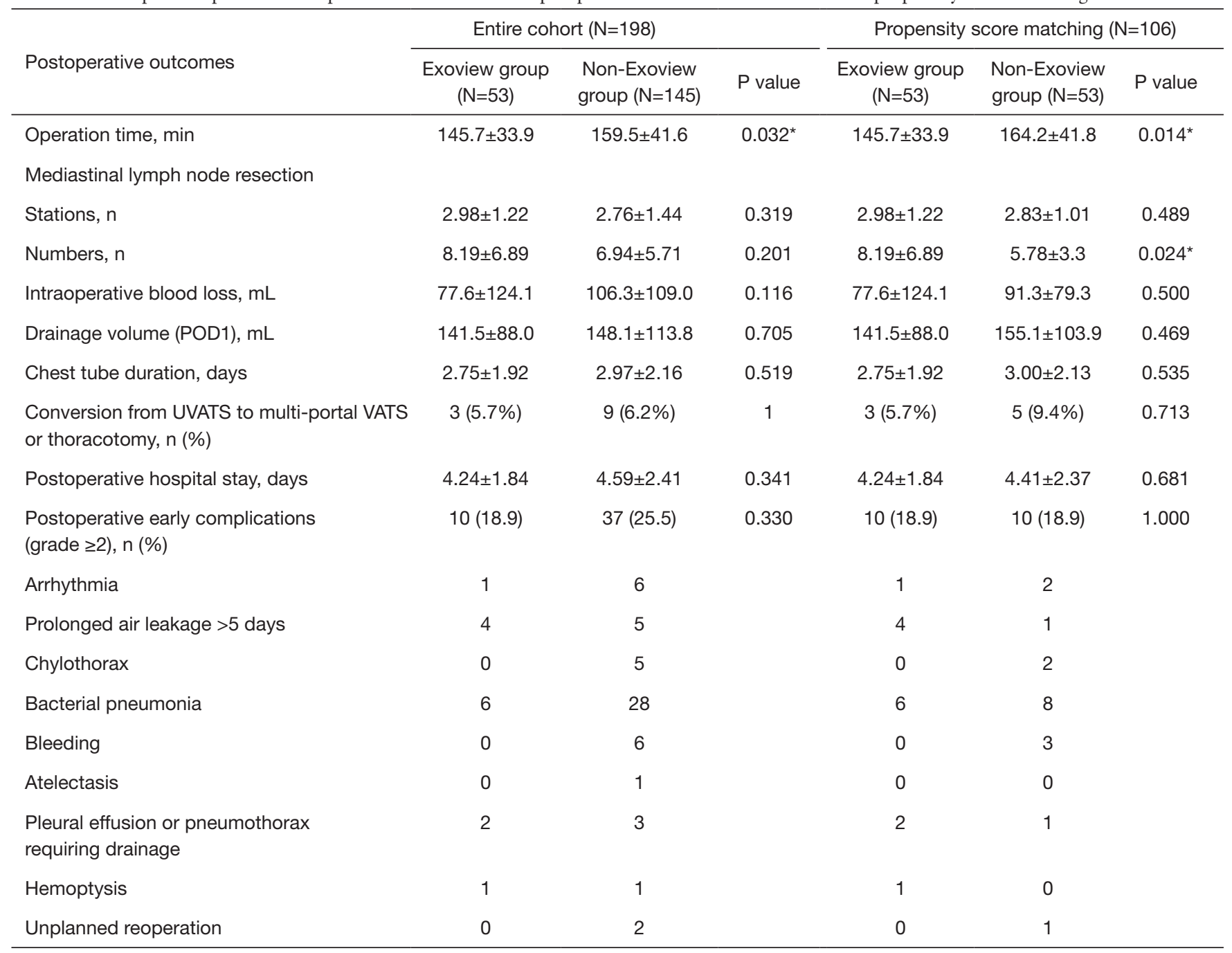

*, P<0.05. POD1, post-operation day 1; UVATS, uniportal video-assisted thoracic surgery.

\section{Arterial variation}

As shown in Figure 3, we found in one of the patients that 2 ascending arteries $\left(\mathrm{A}^{2}\right)$ formed the common truncus with the dorsal artery $\left(\mathrm{A}^{6}\right)$, which normally arises from the right truncus intermedius individually. As shown in Figure 4 of another patient, an extra lateral segmental artery $\left(\mathrm{A}^{4 *}\right)$ beside the normal right middle lobar artery $\left(\mathrm{A}^{4+5}\right)$ flowed into the basal pulmonary artery $\left(\mathrm{A}^{7-10}\right)$. We also detected an extra ascending artery $\left(\mathrm{A}^{1+2} \mathrm{~b}\right)$ branching from the truncus intermedius between $\mathrm{A}^{1+2} \mathrm{a}+\mathrm{b}$ and $\mathrm{A}^{1+2} \mathrm{c}$ in the left upper lobe in 1 patient (Figure 5). Finally, a mediastinal A8 branching from the left main pulmonary artery and running between the superior pulmonary vein and the bronchus was observed in another patient (Figure 6).

\section{Venous variation}

In 1 patient, we observed a $\mathrm{V}^{4}$ that formed a common truncus with $\mathrm{V}^{3} \mathrm{a}-\mathrm{c}$ and flowed into the right upper lobe as shown in Figure 7. In another patient, we also detected a $\mathrm{V}^{6}$ and a common basal vein, which normally form a common truncus, flowing separately into the right lower lobe (Figure 8).

\section{Discussion}

In recent years, UVATS has been adopted by an increasing 
Table 3 Clinical baseline characteristics of patients who underwent single-direction lobectomy with and without preoperative 3D-CTBA

\begin{tabular}{|c|c|c|c|}
\hline Characteristics & \multicolumn{2}{|c|}{ Subgroup cohort $(\mathrm{N}=74)$} & $P$ value \\
\hline Sex, n (\%) & & & 0.270 \\
\hline Male & $9(34.6)$ & $23(47.9)$ & \\
\hline Female & $17(65.4)$ & $25(52.1)$ & \\
\hline Body mass index, $\mathrm{kg} / \mathrm{m}^{2}$ & $24.0 \pm 2.9$ & $24.4 \pm 3.6$ & 0.592 \\
\hline Smoking history, n (\%) & & & 1.000 \\
\hline Never & $21(80.8)$ & $40(83.3)$ & \\
\hline Current/former & $5(19.2)$ & $8(16.7)$ & \\
\hline$\% \mathrm{MVV}, \%$ & $85.6 \pm 18.5$ & $87.6 \pm 16.8$ & 0.640 \\
\hline \multicolumn{4}{|l|}{ Comorbidity, n (\%) } \\
\hline Hypertension & $10(38.5)$ & $18(37.5)$ & 0.935 \\
\hline Diabetes mellitus & $2(7.7)$ & $4(8.3)$ & 1.000 \\
\hline Coronary heart disease & $3(11.5)$ & $8(16.7)$ & 0.803 \\
\hline Maximal lesion size, $\mathrm{mm}$ & $18.4 \pm 7.0$ & $20.5 \pm 12.7$ & 0.445 \\
\hline \multicolumn{4}{|l|}{ Lesion location, n (\%) } \\
\hline Left side & $12(46.2)$ & $14(29.2)$ & 0.144 \\
\hline
\end{tabular}

3D-CTBA, three-dimensional computed tomography bronchography and angiography; FEV1, forced expiratory volume in 1 s; \%FEV1, percentile forced expiratory volume in $1 \mathrm{~s} ; \% \mathrm{MVV}$, percentile maximal voluntary ventilation.

number of surgeons all over the world. This technique, which was first described in 2011, has a faster recovery time and superior cosmetic results compared to conventional multiportal VATS (1). Further, researchers have reported the safety and feasibility of this single incision approach (4-6,22), although no prospective clinical trial has demonstrated a significant difference in operative outcomes between UVATS and traditional multiport VATS. It is well known that UVATS lobectomy constitutes a surgical challenge, even for experienced multiport VATS surgeons, due to the reduced number of operating ports and limited operating space (23). When performing single-port surgery, surgeons are forced to leave their comfort zone and to change some of their surgical habits. Thus, accurate identification and appropriate handling of pulmonary anatomical structures appear to be critical to performing the operation successfully.

$3 \mathrm{D}$ imaging technology has developed rapidly in recent years. With the increasing popularity of anatomical segmentectomy, various $3 \mathrm{D}$ reconstruction software designed to assist with the conversion of $2 \mathrm{D}-\mathrm{CT}$ images to 3D digital images have been developed $(16,17,24)$. Studies 
Table 4 Postoperative parameters of patients who underwent single-direction lobectomy with and without preoperative 3D-CTBA

\begin{tabular}{|c|c|c|c|}
\hline Postoperative outcomes & \multicolumn{2}{|c|}{ Entire subgroup cohort $(\mathrm{N}=74)$} & $P$ value \\
\hline Operation time, min & $140.2 \pm 34.4$ & $157.9 \pm 43.5$ & 0.078 \\
\hline \multicolumn{4}{|l|}{ Lymph node resection } \\
\hline Stations, $\mathrm{n}$ & $3.00 \pm 1.30$ & $2.56 \pm 1.54$ & 0.223 \\
\hline Intraoperative blood loss, $\mathrm{mL}$ & $60.4 \pm 45.4$ & $100.8 \pm 83.9$ & $0.009^{\star}$ \\
\hline Drainage volume (POD1), $\mathrm{mL}$ & $144.6 \pm 83.7$ & $160.6 \pm 134.8$ & 0.584 \\
\hline Chest tube duration, days & $2.96 \pm 2.25$ & $2.77 \pm 1.43$ & 0.658 \\
\hline Conversion from UVATS to multi-portal VATS or thoracotomy, n (\%) & $1(3.8)$ & $2(4.2)$ & 1.000 \\
\hline Arrhythmia & 0 & 2 & \\
\hline Prolonged air leakage $>5$ days & 2 & 0 & \\
\hline Chylothorax & 0 & 2 & \\
\hline Bacterial pneumonia & 4 & 13 & \\
\hline Bleeding & 0 & 2 & \\
\hline Atelectasis & 0 & 0 & \\
\hline Pleural effusion or pneumothorax requiring drainage & 2 & 0 & \\
\hline Hemoptysis & 0 & 1 & \\
\hline
\end{tabular}

*, P<0.05. POD1, post-operation day 1; 3D-CTBA, three-dimensional computed tomography bronchography and angiography.
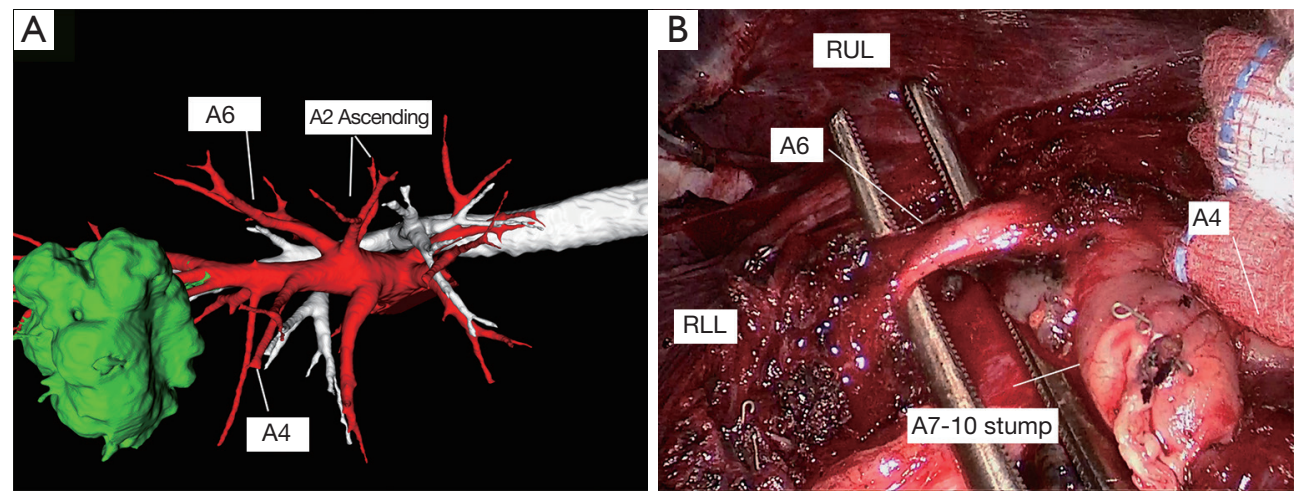

Figure 3 Variation of $\mathrm{A}^{6}$ in right lower lobe. (A) A preoperative $3 \mathrm{D}$ reconstruction of the right pulmonary anatomic structure. The $\mathrm{A}^{6}$ can be seen forming a common truncus with 2 ascending A. (B) Corresponding intraoperative image. In this patient, a right lower lobectomy was performed, so we did not dissect the upper lobar structure further. 3D, three-dimensional; RUL, right upper lobe. 

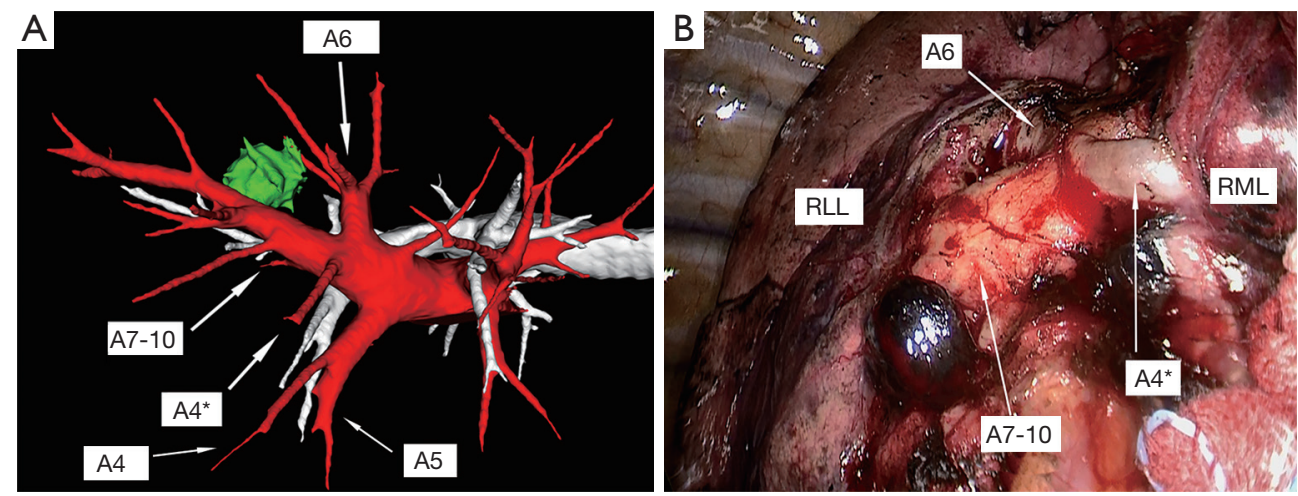

Figure 4 Variation of $\mathrm{A}^{4}$ in right middle lobe. (A) A preoperative 3D reconstruction of the right pulmonary anatomic structure. An extra $\mathrm{A}^{4 *}$ arising from the inferior pulmonary artery can be seen. (B) Corresponding intraoperative image. In this patient, a right lower lobectomy was performed and the $\mathrm{A}^{4 *}$ was preserved according to the preoperative imaging. 3D, three-dimensional; RML, right middle lobe; RLL, right lower lobe.
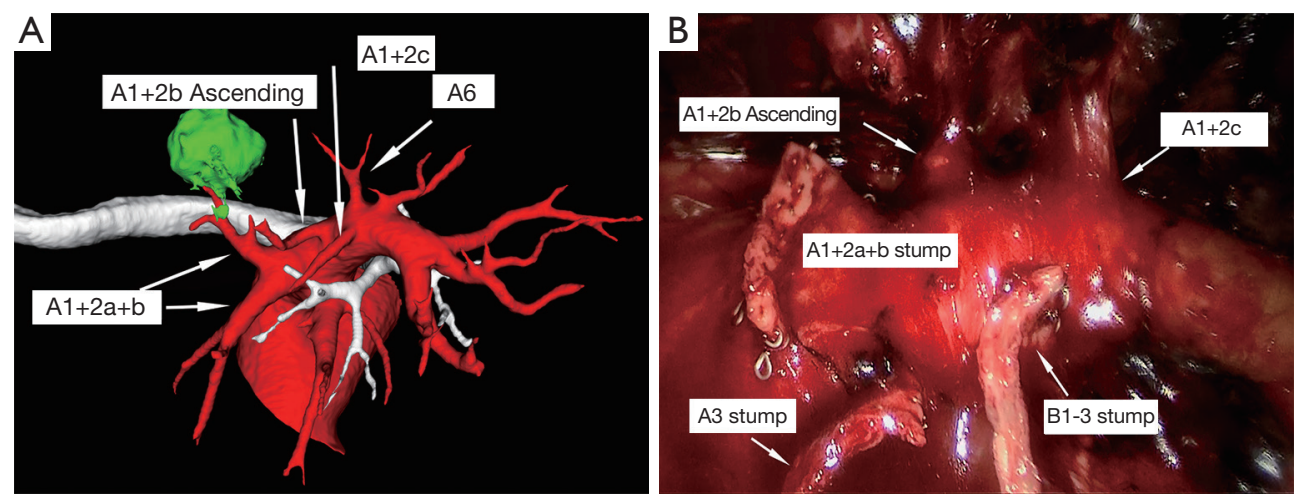

Figure 5 Variation of $\mathrm{A}^{1+2} \mathrm{~b}$ in left upper lobe. (A) A preoperative 3D reconstruction of the left pulmonary anatomic structure. An ascending $\mathrm{A}^{1+2} \mathrm{~b}$ arising from the truncus intermedius can be seen. (B) Corresponding intraoperative image. In this patient, a left upper lobectomy was performed. 3D, three-dimensional.


Figure 6 Variation of $\mathrm{A}^{8}$ in left lower lobe. (A) A preoperative 3D reconstruction of the left pulmonary anatomic structure. A mediastimal $\mathrm{A}^{8}$ branching from the main pulmonary artery can be seen. (B) Corresponding intraoperative image. In this patient, a left lower lobectomy was performed. 3D, three-dimensional; LLL, left lower lobe; IPB, inferior pulmonary bronchus; IPA, inferior pulmonary artery; IPV, inferior pulmonary vein. 

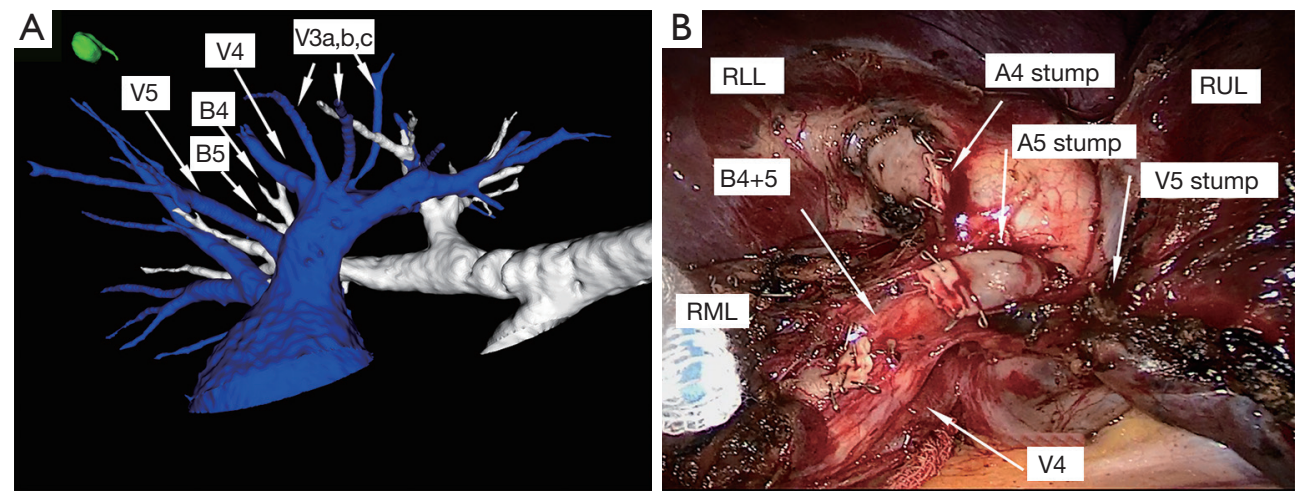

Figure 7 Variation of V4 in right middle lobe. (A) A preoperative $3 \mathrm{D}$ reconstruction of the right pulmonary anatomic structure. A $\mathrm{V}^{4}$ can be seen forming a common truncus with $\mathrm{V}^{3} \mathrm{a}$-c. (B) Corresponding intraoperative image. In this patient, a right middle lobectomy was performed. The $\mathrm{V}^{4}$ and $\mathrm{V}^{5}$ were managed separately during the operation. 3D, three-dimensional. RUL, right upper lobe. RML, right middle lobe. RLL, right lower lobe.
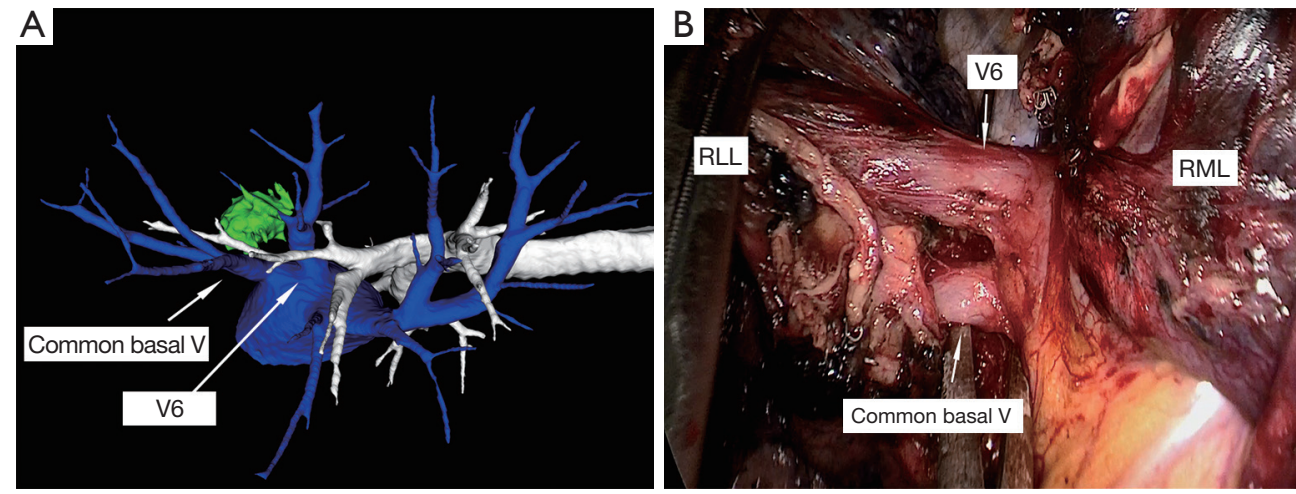

Figure 8 Variation of $\mathrm{V}^{6}$ in right lower lobe. (A) A preoperative 3D reconstruction of the right pulmonary anatomic structure. The $\mathrm{V}^{6}$ and the common basal vein can be seen flowing into the right lower lobe separately. (B) Corresponding intraoperative image. In this patient, a right lower lobectomy was performed. 3D, three-dimensional; RML, right middle lobe; RLL, right lower lobe.

have found $3 \mathrm{D}$ reconstruction to be useful for identifying intersegmental veins as boundary lines of pulmonary segments in the determination of surgical margins before segmentectomy $(17,25)$. To our knowledge, this is the first report to assess the effectiveness of three-dimensional computed tomography bronchography and angiography (3D-CTBA) in UVATS lobectomy based on short-term surgical outcomes. Hagiwara et al. once reported that highquality 3D-CT images were significantly associated with shorter operative time (26). Similarly, our results showed that patients who underwent preoperative $3 \mathrm{D}$ reconstruction tended to have a significantly shorter operative time and a higher number of mediastinal lymph nodes dissected than those without $3 \mathrm{D}$ imaging, suggesting that personalized 3D digital models do contribute to the short-term outcomes of
UVATS lobectomy. Therefore, our study provides a new direction for the application of 3D imaging and facilitates uniportal thoracoscopic anatomic lobectomy, shortening operation time. However, although patients in the Exoview group appeared to have a slight advantage in regard to other intraoperative and postoperative characteristics, there were no significant differences between the two groups. Possible reasons to explain our results are that all the treating surgeons of this cohort have a wealth of experience in thoracic surgery, and it would be difficult to achieve significant improvements in all aspects during the surgery.

Single-direction thoracoscopic lobectomy is a practical surgical technique (20). It overcomes the difficulty in manipulating incomplete lung fissures, as lobectomy is performed progressively in a single direction from 


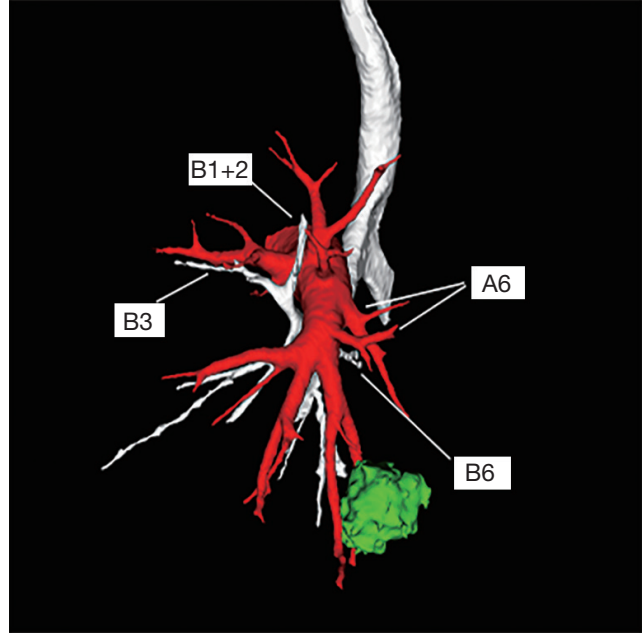

Figure 9 Preoperative simulation of 1 patient with high-positioned A6. In this patient, a left lower lobectomy was performed. The preoperative $3 \mathrm{D}$ reconstruction shows 2 high-positioned $\mathrm{A}^{6}$. Consequently, the incision was performed along the anterior axillary line at the $4^{\text {th }}$ intercostal space instead of the $5^{\text {th }}$ intercostal space. $3 \mathrm{D}$, three-dimensional.

superficial to deep structures. Experience over recent years has demonstrated that this approach is feasible and can simplify the procedure to some extent $(27,28)$. The results of our subgroup analysis of patients who received a single-direction lobectomy show that patients with $3 \mathrm{D}$ imaging had significantly less intraoperative blood loss than those without $3 \mathrm{D}$ reconstruction. This difference may be attributable to this surgical technique requiring physicians to handle pulmonary structures in the lung hilum, which means surgical operation will become quite intractable and dangerous in the event of accidental injury to the blood vessels. Thus, detailed preoperative planning and $3 \mathrm{D}$ visualization imaging which can display the spatial relationship of each branch of the pulmonary vessels helps to avoid blind dissection of vessels, thus reducing the risk of blood loss.

Using preoperative 3D-CTBA, we accurately identified most pulmonary vessel branches and confirmed all aberrant $\mathrm{PA}$ and PV branching patterns related to the surgery with precision. Other researchers have also studied uncommon $\mathrm{PA}, \mathrm{PV}$, or bronchial variations in surgery using $3 \mathrm{D}$ imaging (7-11,19). However, most of them focused on the anatomical pathway of the anomalous vein, and their research mostly involved anatomical segmentectomy. Furthermore, while venous variations are relatively more common and protean, our study concentrated more on the aberrant patterns of pulmonary arteries, because there is a greater risk of arterial bleeding than that of pulmonary veins. Moreover, the pulmonary vein trunk is often stapled at the base when performing a lobectomy unless it flows into another lobe as we described (Figures 7,8).

Preoperative imaging can also help with the selection of surgical incision. It is worth noting that many patients have a superior artery $\left(\mathrm{A}^{6}\right)$ arising from a high location on the left side (a representative image is shown in Figure 9). Based on our clinical experience, performing a UVATS lobectomy through a single incision at the $5^{\text {th }}$ intercostal space can prove challenging in these patients, and adjusting the incision to the $4^{\text {th }}$ intercostal space can make the operation easier to perform. Furthermore, it is sometimes difficult to identify cases of a high-positioned $\mathrm{A}^{6}$ and ascending $\mathrm{A}^{2}$ using $3 \mathrm{D}$ imaging. Therefore, if a patient has a poorly developed lung fissure, the surgeon should combine 2D images with $3 \mathrm{D}$ reconstruction and operate extremely carefully.

With our accumulation of clinical experience using Exoview software, several advantages of this $3 \mathrm{D}$ digital model have become apparent. Firstly, a young physician without expert knowledge of synthetic imaging and pulmonary anatomy can easily reconstruct a $3 \mathrm{D}$ simulation of each patient. After the physician becomes familiar with the system, the mean processing time is approximately 15 minutes. 3D CTBA provides stereo perception and observation of the pulmonary anatomical structures. Therefore, it can be used for preoperative simulation, which can help surgeons to quickly understand the pulmonary anatomy, shortening their learning curve and enhancing their confidence to perform the surgery, especially for young, less experienced surgeons $(19,29)$. Secondly, Exoview is free and open-source software (both the download link and guidebook can be obtained by contacting the corresponding author by email). Furthermore, the reconstruction is free for the patient, so it will not increase their medical costs. Based on our experience and research results, it is essential to make a careful preoperative plan before performing the anatomical lobectomy with UVATS, including the assessment of pulmonary structures and selection of operation pathway. In addition to a huge amount of surgical training, $3 \mathrm{D}$ reconstruction may be a more efficient way to help thoracic surgeons be familiar with anatomical knowledge and overcome technical challenges. Thus, we recommend especially young thoracic 

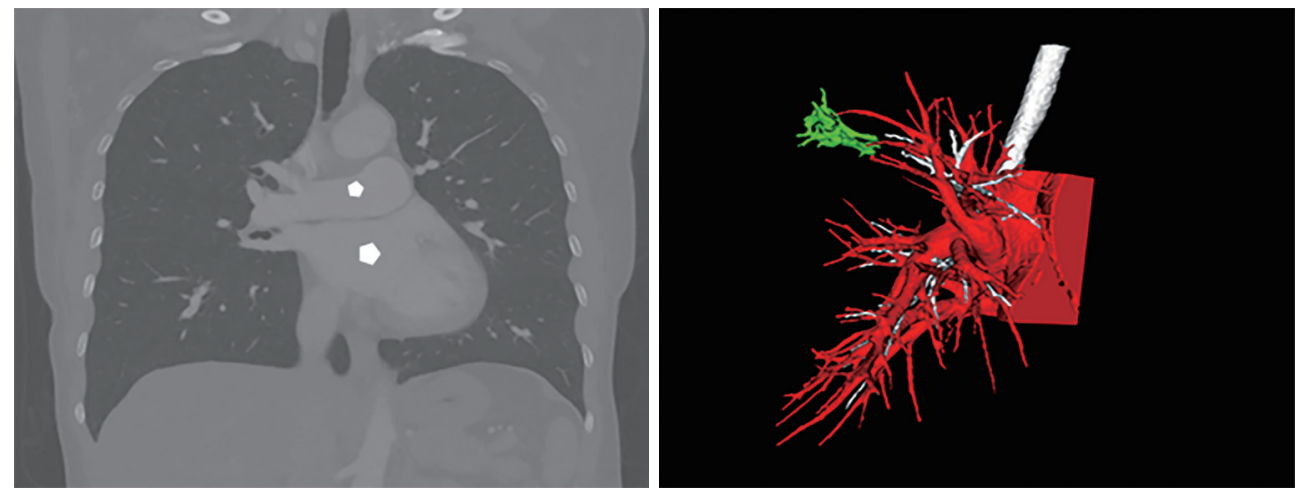

Figure 10 A preoperative 3D reconstruction of the right pulmonary anatomic structure deemed unsatisfactory due to the poor quality of the enhanced CT scan. 3D, three-dimensional.
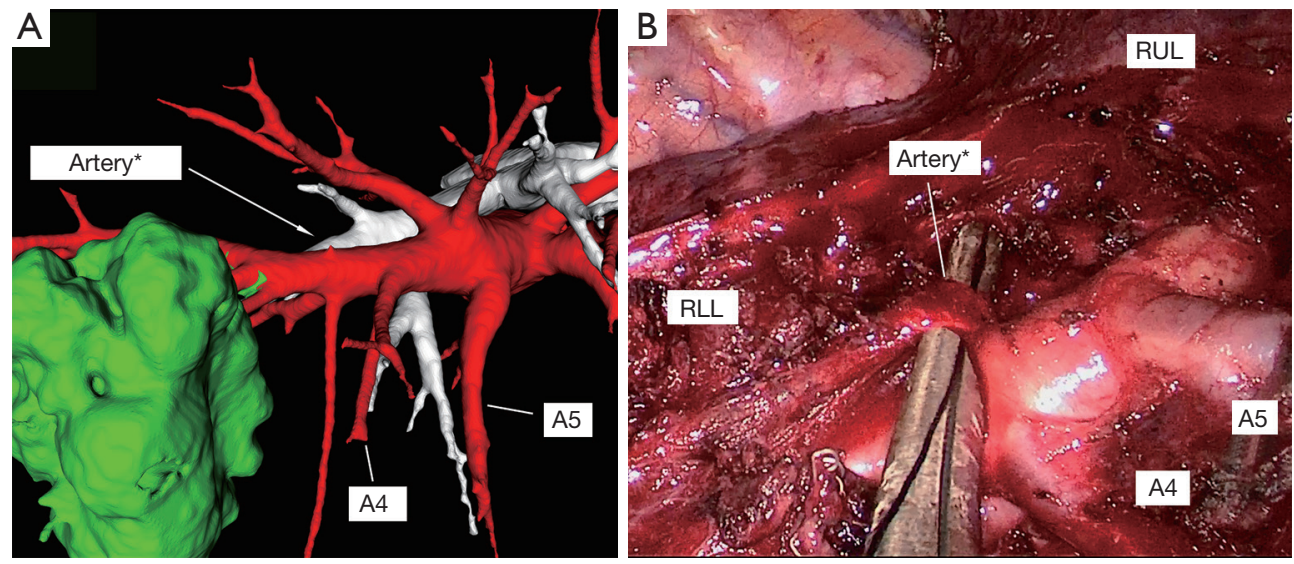

Figure 11 Preoperative simulation and intraoperative control image mismatched in 1 patient. (A) A preoperative 3D reconstruction of the right pulmonary anatomic structure. In this patient, a right lower lobectomy was performed. No branches of the basal artery are visible. (B) Corresponding intraoperative image. The intraoperative findings in this patient showed a 2-mm small artery branching from the basal artery. 3D, three-dimensional; RUL, right upper lobe; RLL, right lower lobe.

surgeons to master preoperative three-dimensional (3D) reconstruction.

There are two main disadvantages of $3 \mathrm{D}$ reconstruction with Exoview. Firstly, as described above, patients must undergo a specialized enhanced CT (CTPA/V). There are also certain requirements for $\mathrm{CT}$ image quality, which can be affected by many factors, such as the experience of the radiologist and the status of the patient's breath hold. As illustrated in Figure 10, the 3D simulation cannot be constructed effectively if contrast medium is full of pulmonary arteries and veins simultaneously. Thus, we always invited the same specialized experienced radiologist Fei-rong Yao to perform the CTPA/V. Secondly, the reconstructions obtained may be distorted as a result of the secondary processing, and some small branches of the pulmonary vessels (which usually refers to those measuring 1-2 $\mathrm{mm}$ ) may be missed. A representative image is shown in Figure 11. Therefore, it is necessary to combine 2D CT with $3 \mathrm{D}$ reconstruction before surgery.

The limitations of this cohort study include but are not limited to its small sample size and potential bias stemming from its retrospective nature. Although we performed a PSM analysis to minimize the influence of selection bias, other potential biases may have had an influence on the short-term benefits such as operative time and adverse events. A prospective randomized trial and long-term follow-up are needed to further confirm the benefits of using $3 \mathrm{D}$ software preoperatively. 


\section{Conclusions}

In summary, this study demonstrated that preoperative 3D-CTBA with Exoview for the evaluation of pulmonary structures was beneficial to performing UVATS anatomical lobectomy safely and effectively. Patients who underwent $3 \mathrm{D}$ imaging had a shorter operation time than those who did not, which might lead to enhanced recovery after surgery. Further advances in 3D imaging technology will not only improve surgical outcomes, but will also assist in the anatomic education and training of young physicians.

\section{Acknowledgments}

Funding: This work was supported by the grants from National Natural Science Foundation of China (No. 81873417 ) and Suzhou Government in China (No. LCZX201301).

\section{Footnote}

Reporting Checklist: The authors have completed the STROBE reporting checklist. Available at http://dx.doi. org/10.21037/atm-21-474

Data Sharing Statement: Available at http://dx.doi. org/10.21037/atm-21-474

Conflicts of Interest: All authors have completed the ICMJE uniform disclosure form (available at http://dx.doi. org/10.21037/atm-21-474). The authors have no conflicts of interest to declare.

Ethical Statement: The authors are accountable for all aspects of the work in ensuring that questions related to the accuracy or integrity of any part of the work are appropriately investigated and resolved. This retrospective study was approved by The First Affiliated Hospital of Soochow University (ethical approval No. 2020232). All data were treated anonymously to protect personal privacy. All procedures performed in this study involving human participants were in accordance with the Declaration of Helsinki (as revised in 2013). Individual consent for this retrospective analysis was waived.

Open Access Statement: This is an Open Access article distributed in accordance with the Creative Commons Attribution-NonCommercial-NoDerivs 4.0 International
License (CC BY-NC-ND 4.0), which permits the noncommercial replication and distribution of the article with the strict proviso that no changes or edits are made and the original work is properly cited (including links to both the formal publication through the relevant DOI and the license). See: https://creativecommons.org/licenses/by-nc-nd/4.0/.

\section{References}

1. Gonzalez D, Paradela M, Garcia J, et al. Single-port videoassisted thoracoscopic lobectomy. Interact Cardiovasc Thorac Surg 2011;12:514-5.

2. Sihoe ADL. Uniportal Lung Cancer Surgery: State of the Evidence. Ann Thorac Surg 2019;107:962-72.

3. Wu CF, Fernandez R, de la Torre M, et al. Midterm survival outcome of single-port video-assisted thoracoscopic anatomical lung resection: a two-centre experience. Eur J Cardiothorac Surg 2018;54:252-9.

4. Xu GW, Xie MR, Wu HR, et al. A prospective study examining the impact of uniportal video-assisted thoracic surgery on the short-term quality of life in patients with lung cancer. Thorac Cancer 2020;11:612-8.

5. Bin Yameen TA, Gupta V, Behzadi A. Uniportal versus multiportal video-assisted thoracoscopic surgery in the treatment of lung cancer: a Canadian single-centre retrospective study. Can J Surg 2019;62:468-74.

6. Wang L, Liu D, Lu J, et al. The feasibility and advantage of uniportal video-assisted thoracoscopic surgery (VATS) in pulmonary lobectomy. BMC Cancer 2017;17:75.

7. Fourdrain A, De Dominicis F, Blanchard C, et al. Threedimensional CT angiography of anatomic variations in the pulmonary arterial tree. Surg Radiol Anat 2018;40:45-53.

8. Murota M, Yamamoto Y, Satoh K, et al. An analysis of anatomical variations of the left pulmonary artery of the interlobar portion for lung resection by three-dimensional CT pulmonary angiography and thin-section images. Jpn J Radiol 2020;38:1158-68.

9. Nagashima T, Shimizu K, Ohtaki Y, et al. Analysis of variation in bronchovascular pattern of the right middle and lower lobes of the lung using three-dimensional CT angiography and bronchography. Gen Thorac Cardiovasc Surg 2017;65:343-9.

10. Nakamura A, Okumura Y, Hashimoto M, et al. Right Lower Lobectomy for an Aberrant Mediastinal Inferior Lobar Artery. Ann Thorac Surg 2020;109:e415-7.

11. Shimizu K, Nagashima T, Ohtaki Y, et al. Analysis of the variation pattern in right upper pulmonary veins and establishment of simplified vein models for 
anatomical segmentectomy. Gen Thorac Cardiovasc Surg 2016;64:604-11.

12. Akiba T, Marushima H, Kamiya N, et al. Thoracoscopic lobectomy for treating cancer in a patient with an unusual vein anomaly. Ann Thorac Cardiovasc Surg 2011;17:501-3.

13. Cui Z, Ding C, Li C, et al. Preoperative evaluation of the segmental artery by three-dimensional image reconstruction vs. thin-section multi-detector computed tomography. J Thorac Dis 2020;12:4196-204.

14. She XW, Gu YB, Xu C, et al. Three-dimensional (3D)computed tomography bronchography and angiography combined with 3D-video-assisted thoracic surgery (VATS) versus conventional 2D-VATS anatomic pulmonary segmentectomy for the treatment of non-small cell lung cancer. Thoracic Cancer 2018;9:305-9.

15. Nakamoto K, Omori K, Nezu K, et al. Superselective segmentectomy for deep and small pulmonary nodules under the guidance of three-dimensional reconstructed computed tomographic angiography. Ann Thorac Surg 2010;89:877-83.

16. Wu WB, Xu XF, Wen W, et al. Three-dimensional computed tomography bronchography and angiography in the preoperative evaluation of thoracoscopic segmentectomy and subsegmentectomy. J Thorac Dis 2016;8:S710-5.

17. Xu G, Chen C, Zheng W, et al. Application of the IQQA$3 \mathrm{D}$ imaging interpretation and analysis system in uniportal video-assisted thoracoscopic anatomical segmentectomy: a series study. J Thorac Dis 2019;11:2058-66.

18. Xue L, Fan H, Shi W, et al. Preoperative 3-dimensional computed tomography lung simulation before video-assisted thoracoscopic anatomic segmentectomy for ground glass opacity in lung. J Thorac Dis 2018;10:6598-605.

19. Zhang $M$, Liu D, Wu W, et al. Preoperative 3D-CT bronchography and angiography facilitates single-direction uniportal thoracoscopic anatomic lobectomy. Ann Transl Med 2019;7:526.

20. Liu L, Che G, Pu Q, et al. A new concept of endoscopic lung cancer resection: Single-direction thoracoscopic lobectomy. Surg Oncol 2010;19:e71-7.

21. Dindo D, Demartines N, Clavien PA. Classification of

Cite this article as: Zhu XY, Yao FR, Xu C, Ding C, Chen J, Wang WY, Pan LY, Zhao J, Li C. Utility of preoperative three-dimensional CT bronchography and angiography in uniportal video-assisted thoracoscopic anatomical lobectomy: a retrospective propensity score-matched analysis. Ann Transl Med 2021;9(6):480. doi: 10.21037/atm-21-474

\section{Zhu et al. Utility of preoperative 3D-CTBA in UVATS lobectomy}

surgical complications: a new proposal with evaluation in a cohort of 6336 patients and results of a survey. Ann Surg 2004;240:205-13.

22. Xie D, Wang H, Fei K, et al. Single-port video-assisted thoracic surgery in 1063 cases: a single-institution experiencedagger. Eur J Cardiothorac Surg 2016;49 Suppl 1:i31-6.

23. Andrade R. Commentary: Why do uniportal video-assisted thoracoscopic lobectomy? J Thorac Cardiovasc Surg 2020;159:2496-7.

24. Wu WB, Xia Y, Pan XL, et al. Three-dimensional navigation-guided thoracoscopic combined subsegmentectomy for intersegmental pulmonary nodules. Thorac Cancer 2019;10:41-6.

25. Oizumi H, Kanauchi N, Kato H, et al. Anatomic thoracoscopic pulmonary segmentectomy under 3-dimensional multidetector computed tomography simulation: a report of 52 consecutive cases. J Thorac Cardiovasc Surg 2011;141:678-82.

26. Hagiwara M, Shimada Y, Kato Y, et al. High-quality 3-dimensional image simulation for pulmonary lobectomy and segmentectomy: results of preoperative assessment of pulmonary vessels and short-term surgical outcomes in consecutive patients undergoing video-assisted thoracic surgerydagger. Eur J Cardiothorac Surg 2014;46:e120-6.

27. Feng $M$, Lin $M$, Shen $Y$, et al. Uniportal video-assisted thoracic surgery for left upper lobe: single-direction lobectomy with systematic lymphadenectomy. J Thorac Dis 2016;8:2281-3.

28. Lin XM, Yang Y, Chi C, et al. Video-assisted thoracoscopic lobectomy: single-direction thoracoscopic lobectomy. J Thorac Dis 2013;5:716-20.

29. Qiu B, Ji Y, He H, et al. Three-dimensional reconstruction/personalized three-dimensional printed model for thoracoscopic anatomical partial-lobectomy in stage I lung cancer: a retrospective study. Transl Lung Cancer Res 2020;9:1235-46.

(English Language Editor: J. Reynolds) 